Universidad Nacional de La Plata

Facultad de Humanidades y Ciencias de la Educación

Departamento de Sociología

\title{
El análisis sociológico en el agro pampeano: apuntes conceptuales para el abordaje de las relaciones sociales entre actores en el trabajo agropecuario
}

\author{
The sociological analysis in the pampeano agro: conceptual notes \\ for the approach of the social relations between actors in the \\ agricultural work
}

\section{Manuela Moreno *}

* Universidad Nacional de Quilmes, Centro de Investigación en Economía y Sociedad de la Argentina Contemporánea-CONICET, Argentina | manuelamoreno.Is@gmail.com

\section{PALABRAS CLAVE}

Relaciones sociales

Agro pampeano

Categorías conceptuales

KEYWORDS

Social relations

Pampeano agro

Conceptual categories

\section{RESUMEN}

El artículo presenta una propuesta de abordaje conceptual para el análisis de las relaciones sociales en el trabajo agropecuario pampeano. Además de introducir brevemente el tema del agro, las relaciones sociales y el abordaje sociológico, el artículo presenta una serie de dimensiones que se consideran centrales para dicho estudio, a partir de los conceptos de autores con diversas trayectorias teóricas dentro de las ciencias sociales. Dentro de cada una de las dimensiones, se describen cuáles son las categorías relevantes y los interrogantes que podemos generar a partir de ellas en el estudio concreto de las relaciones sociales en el agro. Finalmente, se esboza, a modo de propuesta, la forma en que consideramos la articulación entre dimensiones y categorías conceptuales, dando lugar a la configuración de tipos opuestos de relaciones sociales y el modo en que los consideraremos en el estudio empírico.

\section{ABSTRACT}

The article presents a proposal of conceptual approach for the analysis of the social relations in the agricultural work pampeano. In addition to briefly introducing the theme of agriculture, social relations and sociological approach, the article presents a series of dimensions considered central to this study, based on the concepts of authors with diverse theoretical trajectories within the social sciences. Inside each of the dimensions, we describe what are the relevant categories and the questions that we can generate from them in the concrete study of social relations in agriculture. Finally, we propose, as a proposal, the way we consider the articulation between dimensions and conceptual categories giving rise to the configuration of opposing types of social relations and the way in which we will consider them in the empirical study.

Fecha de recibido: 11/05/2017 | Fecha de aceptado: 24/08/2017 | Fecha de publicado: 28/12/2017

Cita sugerida: Moreno, M. (2017). El análisis sociológico en el agro pampeano: apuntes conceptuales para el abordaje de las relaciones sociales entre actores en el trabajo agropecuario. Cuestiones de Sociología, 17, e039. 


\section{INTRODUCCION}

Históricamente el agro pampeano ha tenido una centralidad en nuestro país, tanto a nivel de generación de ingresos y divisas como desde el punto de vista social y político. La conformación de buena parte de los pueblos y ciudades intermedias del interior de las provincias que componen la región pampeana ha estado signada por la inmigración europea asociada al desarrollo agropecuario desde medianos/fines del siglo XIX, constituyendo el "campo" un referente social portador de una serie de significados. Recorriendo los pueblos y ciudades intermedias de la provincia de Buenos Aires se puede percibir cierto imaginario social asociado a frases como "acá todos vivimos del campo", “en el campo nos conocemos todos”, que ponen de manifiesto el rol simbólico que tiene en los espacios locales, como sector económico y como fuente de relaciones personales con características particulares. Una construcción con fuerte carga de idealización o visión romántica sobre el sector, en la cual las características de las relaciones sociales (opuestas a las de otros sectores de la economía) es central. Se trata de un tema abordado en estudios clásicos de las ciencias sociales, pero también una discursividad presente en el imaginario social sobre el sector (y muy destacado por los mismos actores del agro).

Frente a los cambios ocurridos en las últimas décadas, sobre todo de índole productivos y las nuevas tecnologías, el sector enfrenta hoy una serie de tensiones a nivel de los sentidos históricamente asociados. Esto se convierte en una preocupación de buena parte de sus referentes, que buscan desarrollar estrategias de construcción de una “imagen pública” del campo actual $\stackrel{1}{\text {. Es }}$ decir que, frente a los cambios, los mismos actores del sector también enfrentan una crisis de la forma en que se definen y cómo los identifican socialmente.

En el marco de esta imagen del "campo", las características que tienen las relaciones sociales, entendidas como los vínculos entre los distintos sujetos necesarios para el desarrollo de la producción, es de suma importancia. Cabe preguntarse si se trata de un sector de la economía signado por las relaciones personales, donde "todos se conocen", con fuerte presencia de lo familiar y con rasgos de paternalismo, o si se trata de un sector que cada vez más se parece a otros de la economía, donde priman las relaciones estructuradas por el mercado, la organización científica del trabajo y la impersonalidad. También, en qué medida los cambios económicos, productivos, sociales y subjetivos han transformado las dinámicas sociales en el sector, y si las modificaciones en el tipo de las relaciones sociales resultan condición necesaria para que un agro netamente capitalista se consolide.

Dentro de los estudios que abordan temas agrarios, y específicamente de la región pampeana, coinciden en que a partir de la década de 1970 el agro pampeano viene experimentando una transformación profunda vinculada a aspectos tecnológicos, sociales y de organización de la producción. En algunos casos se trata de un fenómeno vinculado a los procesos de globalización/mundialización (Albadalejo, 2013; Giarraca y Teubal, 2008; Giberti, 2009; Sili, 2005), un avance de las relaciones plenamente capitalistas en el sector (Balsa, 2006), específicamente a la construcción de los agronegocios (Gras y Hernández, 2009 y 2013), una aceleración de tendencias de modernización presente desde los años 1970 (Barsky y Gelman, 2009) o un "cambio de paradigma productivo" (Anlló, Bisang y Campi, 2013). Independientemente de cómo se conceptualice el cambio, los estudios coinciden en que el proceso implicó redefiniciones e incorporaciones en los actores sociales agrarios y sus formas de organizar el trabajo y los modos de 
relacionarse con otros y con el territorio.

Sin embargo, el hecho de que se hayan dado cambios en la producción no implica que todos los actores los desarrollen del mismo modo. Si bien una característica de estas últimas décadas ha sido una tendencia hacia formas más empresariales de producción, no significa que ello haya ocurrido completamente. Mientas algunos actores transformaron o configuraron sus formas de organizar el trabajo bajo nuevas lógicas, otros las mantuvieron (incluso reforzaron el modo en que tradicionalmente lo hacían), y otro tanto reconfiguraron solo algunos aspectos.

Entonces, la preocupación por las formas que asume el trabajo y las relaciones sociales características del sector es un tema presente en diversos análisis sociales sobre el sector. En nuestro caso, nos interesa avanzar en este punto porque, creemos, también puede dar lugar a comprender las formas que adquiere la producción. En este sentido, el interrogante central de trabajo $\underline{\underline{2}}$ es en qué medida los cambios productivos y sociales han reformulado las formas de organizar el trabajo. Preguntarnos, además, si estas redefiniciones implican modificaciones sustanciales de las relaciones sociales características del sector, en el sentido de que prima la separación entre los espacios de vida y los de trabajo, una mayor regulación mercantil de los vínculos, siendo un “factor” mas a conseguir por medio del mercado; o si, en cambio, las relaciones personales y familiares mantienen un rol central en la estructuración del trabajo, manteniendo la combinación histórica entre espacios sociales (personales y laborales), y siendo las características de las relaciones un componente de persistencia en un sector con fuertes transformaciones.

En el presente artículo expondremos las categorías conceptuales que nos resultan ordenadoras del trabajo empírico y que nos permiten una perspectiva propia de cómo abordar las relaciones sociales en el trabajo agropecuario.

Para tal fin incorporamos una serie de autores de posiciones teóricas diferentes (incluso opuestas) y con los cuales no necesariamente acordamos en la totalidad de su desarrollo teórico. Pues la intención es abrir una serie de preguntas sobre el trabajo empírico, apartarnos así de un uso más corriente de la teoría como un marco general donde encuadrar un trabajo particular. La teoría ha sido utilizada aquí en una función heurística (Saltalamacchia, 1994) a fin de generar preguntas (no buscando exclusivamente las respuestas en ella) $\frac{3}{3}$ y tomar conceptos que nos permitan explorar aspectos de nuestro trabajo. Si bien reconocemos el lugar central de las teorías al inicio de toda investigación, creemos que abordar el estudio de las características específicas de un fenómeno social permite regresar sobre las cuestiones teóricas una vez analizado el hecho concreto. Por esta razón, en el presente artículo no se encontrará un desarrollo exhaustivo de una o varias teorías, sino la exposición de una serie de conceptos de diversos autores que nos permitieron identificar y reflexionar sobre aspectos centrales del trabajo empírico.

\section{Dimensiones de análisis de las relaciones sociales}

Dentro de las diversas perspectivas que abordan las dinámicas relacionales del mundo social, existen diferentes formas de definir las relaciones sociales. Mark Granovetter define lo relacional a partir del concepto de vínculos interpersonales, entendidos como la "combinación del tiempo, la intensidad emocional, intimidad (confianza mutua) y los servicios recíprocos que caracterizan a 
dicho vínculo” (Granovetter, 1973, p. 2). Si bien las dimensiones resultan de interés, el problema de este análisis es que los lazos son tratados, como señala el mismo autor, "como positivos y simétricos” (Granovetter, 1973).

Por este motivo, resulta pertinente agregar la distinción que realiza Gutiérrez (2007) sobre los “dos niveles de lo relacional”. Los mismos remiten a una dimensión estructural que incluye condiciones objetivas externas (históricamente situadas) y su relación con aquellos elementos que permiten ubicar a los agentes e instituciones en el espacio social: volumen y estructura de las diferentes especies de capital, que constituyen así las condiciones de posibilidad de la circulación de distintos tipos de bienes y servicios entre los componentes de las redes. También remite a una dimensión vincular, que consiste en el análisis de esas interacciones concretas, de su intensidad, de su frecuencia, del tipo y calidad de los bienes:

Una dimensión vincular, que consiste en el análisis de esas interacciones concretas, de su intensidad, de su frecuencia, del tipo y calidad de los bienes (materiales o nomateriales) y de los servicios que circulan, etc. Aquí es importante incluir en el análisis elementos que hacen a la subjetividad de los agentes implicados, ligados a trayectorias individuales y a experiencias personales (Gutiérrez, 2007, p.15).

Según estos autores mencionados, la cuestión del origen, la frecuencia y los intercambios son centrales para analizar las interacciones en el plano concreto. Teniendo en cuenta esta importancia, al interior de cada una de las explotaciones de nuestra muestra analizaremos la dimensión vincular (dada por el origen, frecuencia y los intercambios) y la forma que adquieren.

Por otro lado, se trata de relaciones sociales en la esfera del trabajo lo que les otorga cierta especificidad. En este sentido, Quaranta (2007), en su análisis sobre los mercados de trabajo y las formas de regulación social, señala que

La transformación de la fuerza de trabajo potencial en un trabajo determinado demanda un complejo proceso de negociación entre los trabajadores y los empresarios que resulta en una combinación de dispositivos de control e instancia de consenso construida social y políticamente a diferentes niveles de la organización social. Estos procesos dependen tanto de lo que sucede en el sitio de producción como en otras esferas de organización social vinculadas al trabajo (Quaranta, 2007, p. 94)

Según el autor, las características de las relaciones sociales (cómo se estructuran, de qué tipo son), la confianza, las formas de control y el involucramiento en el proceso productivo, junto a los sistemas de remuneración resultan variables a tener en cuenta en el análisis de los factores relacionales dentro del mundo laboral. $\stackrel{4}{-}$

En nuestro caso, para describir la forma que adquieren las relaciones en el marco del proceso laboral consideraremos tres cuestiones: cuáles son los soportes sobre los que se anclan las relaciones, qué forma adquiere la confianza y la cuestión de la supervisión en las tareas, y cómo son las formas de intercambio en el marco de las interacciones, destacando, además, las formas que adquieren la remuneración. 
Para describir la estructuración de las relaciones sociales (origen, temporalidad y formas de confianza) tomaremos los planteos realizados por Anthony Giddens en su teoría sobre la modernidad, específicamente las formas de anclaje (o desanclaje y reanclaje) de los vínculos sociales en distintos contextos, y los análisis sobre la temática de la confianza en procesos de transformación social. Para describir los aspectos de los intercambios que se dan en el marco de las relaciones (central para comprenderlas, sobre todo por la naturaleza de las relaciones, en este caso, laborales) a partir de los planteos de autores que se inscriben dentro de las investigaciones de la sociología económica (Florence Weber, Viviana Zelizer). Finalmente, consideraremos la tensión entre formas opuestas de estructurar las relaciones sociales. En este punto señalaremos la tensión entre tipos de racionalidades opuestas y las transformaciones en las relaciones en el marco de los procesos de racionalización de Max Weber. El artículo se desarrolla presentando una breve introducción de las categorías y autores y, seguidamente, cuál es su importancia y qué interrogantes nos permite abordar en el estudio de las relaciones sociales en el trabajo agropecuario. Luego de presentar estas categorías, a modo de reflexión, proponemos una síntesis de las mismas a partir de su articulación en dos formas opuestas de relaciones sociales que hemos construido para el abordaje del trabajo empírico en el agro pampeano.

\subsection{Anclaje de las relaciones sociales en distintos contextos sociales}

Entre los aspectos a considerar en la estructura de las relaciones sociales para resolver el trabajo, tendremos en cuenta sobre todo, tomando los planteos realizados por Giddens, los soportes sobre los cuales se anclan las relaciones en los distintos contextos.

La diferenciación de una forma de estructurar las relaciones sociales, es decir, una forma de organizar lo social, ha sido conceptualizada de diversas formas en términos de diferenciación o de especialización funcional (funcionalismo). En general, los estudios contraponen la idea del carácter comunal de los órdenes tradicionales con la impersonalidad de la vida moderna, la clásica distinción entre comunidad y sociedad de Tönnies.

Giddens opta por el concepto de desanclaje $\underline{5}$, como el "despegar” las relaciones sociales de sus contextos locales de interacción y reestructurarlas en indefinidos intervalos de tiempo y espacio (Giddens, 1995) y "reancladas" sobre otros soportes. Si bien el planteo del autor implica una referencia a esta conceptualización clásica de comunidad y sociedad, señala que es un error contraponer la impersonalidad de lo moderno contra la intimidad de la vida personal en lo tradicional. La vida personal y las relaciones sociales están profundamente ligadas en las interacciones de la modernidad (Giddens, 1997). Además, se aparta de la idea de transición y referencias cronológicas propias de las teorías de la modernización (que explican el pasaje de una forma social a otra).

En este sentido, en la articulación propia de las sociedades premodernas (o tradicionales) lo que predomina es el anclaje de las relaciones en cuatro contextos localizados: el sistema de parentesco, la comunidad local, la religión y la tradición. En nuestro caso, solo consideraremos tres que nos parecen pertinentes desarrollar para el estudio de los actores en el agro.

El sistema de parentesco (aunque no es del mismo orden, incluye también a las relaciones de amistad $^{6}$ ), es un punto que nos interesa particularmente por la relevancia que ha tenido y conserva 
aun hoy en la organización del trabajo en el agro pampeano. Giddens señala que este sistema proporciona un modo relativamente estable de organizar "haces" de relaciones sociales a través del tiempo y del espacio (Giddens, 1993, p. 99). Si bien condensan también una serie de tensiones y conflictos, las relaciones con parientes (independientemente del afecto y la buena o mala relación entre las personas concretas) son dependientes de desarrollar una serie de obligaciones, además de proporcionar una red estable de relaciones íntimas. Es decir, el parentesco provee una serie de nexos sociales fiables que conforman el medio de organizar las relaciones de confianza. Con el avance de la modernidad, las relaciones de parentesco, si bien mantienen su importancia, especialmente dentro del marco de la familia nuclear, dejan de ser las portadoras de los vínculos sociales intensamente organizados a través del espacio-tiempo (Giddens, 1993, p. 105).

El segundo contexto que menciona Giddens es el de la comunidad local. El entorno local es, en estos contextos premodernos, el sitio en el que se desarrolla el entramado de relaciones sociales que, por poseer un ámbito espacial reducido, proporciona solidez en el tiempo. En cambio en la modernidad, aunque pueden continuar teniendo su importancia, los contextos locales fueron transformados en su centralidad por el distanciamiento espacio temporal, fundamentalmente a partir del entretejido entre lo local y lo global. Aunque pueden persistir los sentimientos de apego e identificación con los lugares, han sido desvinculados: "La comunidad local ha dejado de ser un lugar saturado de significados familiares y sabidos por todos, para convertirse, en gran medida, en expresión localmente situada de relaciones distantes” (Giddens, 1993, p. 106).

El otro contexto que nos interesa resaltar es la tradición. Esta refleja una forma particular de estructurar la temporalidad en la que el modo en que se hacían las cosas en el pasado es el medio de organizar el futuro. En la seguridad de que las cosas saldrán de determinado modo porque ya se realizaron así, la tradición contribuye de manera fundamental a la seguridad ontológica en tanto sostiene la confianza en la continuidad del pasado, presente y futuro, y conecta esa confianza con las prácticas sociales rutinarias. El autor plantea que con el avance de la modernidad estos contextos se desdibujan en cuanto a su rol de estructuradores de las relaciones sociales. La tradición se ve claramente minada por la reflexividad de la vida moderna que se alza en directa oposición a ella.

De tal modo, mientras que en los asentamientos premodernos la fiabilidad básica encaja dentro de las relaciones personalizadas de la comunidad, de las relaciones de parentesco y de amistad, con el desarrollo de los sistemas abstractos (característico de la modernidad) la fiabilidad en principios impersonales y anónimos resulta indispensable para la existencia social.

Las dinámicas de la modernidad desconectan las relaciones de confianza y fiabilidad de los contextos locales, familiares y de la tradición. Si bien reaparecen constantemente, no son los soportes sobre los que se estructuran. En este punto el autor resalta que, a diferencia del planteo clásico que retoma a Tönnies, la vida personal y las relaciones sociales involucradas están profundamente ligadas con los sistemas abstractos de mayor alcance. El punto de diferenciación entre una forma y otra es que las relaciones se construyen sobre bases diferentes pero existen puntos de articulación; Giddens da cuenta de cómo los elementos que organizan a una no desaparecen en la otra, sino que se transforman y redefinen.

Tomando esta perspectiva, consideramos la tensión entre formas opuestas de relacionarse basadas 
en diferentes soportes: por un lado en lo local, el sistema de parentesco y la tradición; por el otro, en los sistemas abstractos. Tomando en cuenta este eje se puede describir en las dinámicas de las relaciones concretas qué rol juegan las relaciones familiares; cómo se dan en cada tipo de interacciones; si son la forma central de origen de las relaciones; qué obligaciones y seguridades conllevan estas relaciones familiares, si el hecho de ser familiares implica otras dinámicas en relación a la confianza, a los intercambios económicos, etc. Además, indagar qué rol juegan, en las distintas explotaciones agropecuarias, las relaciones localizadas, circunscriptas a un espacio concreto y con una frecuencia determinada; si tienen relevancia como parte de la estructuración de una forma de relacionarse en las explotaciones, y qué implicancias tiene en las distintas unidades en términos de las relaciones y de la producción concreta. Finalmente, indagar qué lugar tiene el cambio en las unidades concretas, o si la forma en que se hacían las cosas sigue siendo un apoyo fundamental para estructurar las relaciones en el presente.

\subsection{Formas de estructurar la confianza y la supervisión en el sitio de trabajo}

Otro punto importante para comprender las dinámicas relacionales y las implicancias para la organización del trabajo en las unidades concretas, es la cuestión de la confianza. Al respecto podemos formularnos tres interrogantes iniciales: si las relaciones se estructuran a partir de la confianza; qué diferencias se pueden establecer entre distintas formas de confiar, y qué implicancias tiene para las dinámicas sociales y la producción.

Como señala Hillmann (1994), la confianza es una dimensión básica de la acción social, dado que mediante ella se reduce la complejidad, delimitando la capacidad de experimentar, informarse, decidir y orientarse (haciendo más fácil la previsión y evaluación, simplificando la imagen del medio, etc.).

Si bien la confianza está asociada a contextos de intimidad y relaciones personales, es una condición necesaria de la interacción en sociedades más complejas. Uno de los aspectos que distinguen a las sociedades actuales y los contextos de transformación acelerada es la incertidumbre (Giddens, 1997). La volatilidad, fragmentación, complejización y diferenciación en las sociedades actuales demandan de la confianza como base para que la vida de los agentes logre cierto orden y significado (y no quede reducida a la angustia, la ansiedad, o al caos).

La confianza, en estos contextos complejos, tiene una función ordenadora y estabilizadora que puede ser entendida como el valor de la confianza en tanto "reductora de la complejidad” (Luhman, 1996) en entornos de alta incertidumbre. Y una función selectiva que permite reducir la evaluación de todos los emisores; por ende, la selección puede estar sesgada por la evaluación de la confiabilidad. Por ejemplo, en el caso de los empresarios, estos pueden confiar en sus empleados y su capital social para seleccionar candidatos para un puesto o para acceder a cierto bien o servicio, en lugar de abrir la búsqueda que demandaría mucho tiempo y esfuerzo. En otro aspecto, pueden confiar en los trabajadores y evitar la inversión que requiere la supervisión cotidiana de las tareas. Ambas cuestiones implican cierta organización del trabajo e inversiones de capital (social y económico) (Pucci et al., 2006).

Existen diversas formas de confianza que involucran determinadas estructuraciones de las relaciones sociales. Al respecto, Giddens desarrolla cuáles son las formas en que se construye la 
confianza, no solo en contextos reducidos, con comunidad de valores y creencias, sino que también en contextos despersonalizados. Para este autor, la confianza está vinculada a dos tipos de procesos diferentes: los compromisos de presencia mutua, y los compromisos anónimos, asociados a la fe en los sistemas abstractos, característica de las instituciones modernas ${ }^{\underline{Z}}$ (Giddens, 1995).

El punto de diferenciación entre una forma y otra es que las relaciones se construyen sobre bases diferentes: en las condiciones premodernas se basan en las personas, en el tiempo compartido, en la intimidad y la reciprocidad; mientras que en las modernas, se sostienen en la fiabilidad de los sistemas abstractos. Esta forma de confianza (en sistemas abstractos, específicamente en los expertos) se puede traducir en que se genere una estructura basada en la expertise de diversos tipos de actores (antes que una relación directa en dos o más personas, como en los compromisos de presencia).

Giddens aporta ciertos matices sobre la cuestión de la confianza y los sistemas abstractos. Uno de ellos es que la fiabilidad en estos sistemas proporciona la seguridad de la confianza cotidiana, pero nunca puede ofrecer la reciprocidad ni la intimidad que ofrecen las relaciones personales de confianza. Por esto, el autor destaca la importancia de las personas en los "puntos de acceso", que en un sistema determinado se esfuerzan por presentarse como "dignas de confianza” porque proporcionan el vínculo entre la fiabilidad personal y la del sistema abstracto (Giddens, 1993). Esta dinámica puede constituir un punto de explicación sobre la importancia que asignan los actores en el agro a las relaciones personales, que “conozcan” con quienes trabajan. Sin embargo, sobre esta situación cabe indagar en cada caso concreto si este conocimiento personal involucra otros compromisos o acciones adicionales, siendo un aspecto diferenciador de la forma en que se dan las relaciones sociales en el trabajo.

La temática de la confianza y las formas en que se estructuran se relaciona, en los espacios de trabajo, con la problemática del control y el involucramiento de los trabajadores.

Newby (1983) destaca la existencia de distintas articulaciones de las formas de control de los trabajadores. En este sentido, destaca que la relación que se establece entre el tamaño de la fuerza de trabajo, la definición de la situación de trabajo y el modo de control utilizado no es de carácter lineal o directo, sino que intervienen también en su conformación las modalidades de organización laboral y productiva adoptadas por los productores. A partir de esto, diferencia entre unidades productivas que recurren a formas de organización y control del trabajo de carácter burocrático, mientras que otras se basan en un control "tradicional” o paternalista de sus trabajadores.

En sintonía con esta perspectiva, Quaranta (2007) destaca sobre la cuestión de la supervisión concreta de las tareas (que presentan distintas características según el tipo de relaciones sociales en cada empresa) que

El análisis de las formas de control del trabajo en la agricultura diferenció modalidades de disciplinamiento de la mano de obra según el tipo de dispositivos de control utilizado. Por un lado, se encuentran aquellas unidades donde dispositivos externos al trabajador se ponen en funcionamiento en un contexto de burocratización del establecimiento agropecuario y, por otro, aquellas donde la disciplina se obtiene a partir de relaciones de tipo paternalista (Quaranta, 2007, p. 94) 
Cada forma de control se apoya en mecanismos diferentes: en un tipo las relaciones personales, locales, de parentesco juegan un lugar central, generan un involucramiento de los actores y no se requiere de una supervisión constante en los lugares de trabajo. La relación de los trabajadores con sus empleadores se construye a partir del predominio de lazos morales y obligaciones mutuas, aunque desiguales. Mientras que las formas de supervisión basadas en la estructura científica del trabajo adquieren formas burocratizadas de organización y de control, en las que se utilizan niveles intermedios de mando, introduciendo una escala jerárquica de autoridad que favorece la racionalización de las mismas. Esta racionalización se expresa en una mayor formalización de los dispositivos de control que adquieren un carácter impersonal (Quaranta, 2007).

Sin embargo, el proceso de racionalización de la producción agropecuaria no resulta de un modo unívoco. Newby (1983) destaca al respecto que en la agricultura extensiva (que implica una mayor racionalidad económica) las modalidades de control laboral presentes adquieren rasgos difusos y particularistas, que refuerzan las formas tradicionales de autoridad basadas en vínculos personales. El control se construye articulando roles laborales y no laborales a partir de la movilización de las redes sociales en las que se encuentran incluidos los actores participantes.

Por otro lado, la organización del trabajo y las formas de control utilizadas inciden sobre el involucramiento o el compromiso de los trabajadores con su ocupación. En las explotaciones con una organización científica del trabajo se expresa de modo instrumental, vinculada con las características regladas e impersonales del ejercicio del control. Mientras que aquellas con una organización de tipo "tradicional” (con una menor división técnica del trabajo y formas de control paternalistas) las relaciones de los trabajadores con sus empleadores se construyen (de un modo desigual) a partir del predominio de lazos morales y obligaciones mutuas (Quaranta, 2007).

Finalmente, las formas de control e involucramiento de los trabajadores se vinculan con los modos de remuneración (que indagaremos en el siguiente apartado) (Newby, 1983). Mientras que en un caso se estructuran a partir de los acuerdos salariales y parámetros objetivos, en el otro, a los esquemas salariales se le adicionan remuneraciones monetarias y no monetarias, que fortalecen una forma de identificación basada en lazos personales, favoreciendo la desvinculación de los comportamientos de los actores sociales de su posición de clase (Quaranta, 2007).

Analizar si las relaciones sociales se basan en la confianza (y de qué tipo) y las formas que adquiere la supervisión de las tareas asociadas a ellas, no solo nos permite describir las dinámicas sociales alrededor de la explotación, sino plantear algunas implicancias para el desarrollo de la producción, el trabajo y su transformación reciente.

Entonces, bajo estas categorías proponemos analizar si las relaciones sociales que establecen los empresarios con los diferentes actores se sustentan en la confianza; en qué se basa esta confianza; si tiene implicancias para la organización del trabajo, como herramienta para reducir la complejidad, seleccionar y ordenar. También exploraremos si existe una forma de estructurar las relaciones en base a la confianza en los compromisos de presencia, o lo que predomina es confiar en los "sistemas expertos”. Sobre esta última forma, también se puede agregar qué rol juega lo personal como mediaciones o "puntos de acceso" a las formas abstractas; analizar cómo se vincula esta forma de organizar las relaciones sociales en la organización del trabajo: si la confianza opera como 
reductora de la complejidad que provocan los cambios acelerados en el sector; cómo se traduce esta función en términos del trabajo cotidiano, en compromisos tácitos o explícitos, menor supervisión (por lo tanto "ahorro”), delegación completa de la realización de tareas; y si la función selectiva implica ahorro de tiempos de búsqueda y de recambios, imprimiéndole dinamismo a la producción; o si la estructuración requiere de actores y posiciones basadas en una organización científica del trabajo, y por lo tanto mayor inversión en términos de capital económico y social (ampliar las redes para control y acceso a la información).

\subsection{Los tipos de intercambio}

Por otra parte, consideramos una dimensión central en el análisis de las relaciones en los sitios de trabajo: las formas de intercambio que se dan en el marco de las relaciones sociales, cómo se dan los intercambios en la esfera económica entre personas cercanas (familiares, amigos, etc.); también, qué ocurre con los intercambios económicos: si sólo se basan en el criterio del cálculo o si entran en juego otras cuestiones (personales, familiares, culturales, de clase, etc.).

La cuestión de los intercambios económicos y las relaciones sociales ha sido ampliamente debatida. En general, se tiende a separar las distintas esferas, por ejemplo, como señala Max Weber, la "situación de mercado" (la esfera económica) está basada en un cálculo en dinero, es decir, en estimar en dinero todos los bienes y servicios que pueden entrar en el cambio. Esto implica que los bienes no se estiman en su significado de utilidades con respecto al tiempo, el lugar o las personas, sino que se estiman como probabilidad de cambio en dinero para el titular del poder de disposición (Weber, 2012[1922]).

Sin embargo, distintos autores dentro de la sociología económica han problematizado esta visión de las esferas separadas. Al respecto, Florence Weber destaca que la presencia o ausencia de moneda no expresa nada sobre la naturaleza del intercambio. Este constituye un punto relevante porque puede dar lugar a confusiones. Entonces, un primer punto es que es necesario distinguir cuidadosamente el dinero (medio de pago) del mercado (sistema de formación de los precios). A menudo se confunde transacción mercantil con transacción monetaria, naturaleza de la relación (mercantil o personal) con medio de pago (en efectivo o “en especies”). Una transacción como el trueque es una transacción mercantil sin moneda, mientras que pueden existir intercambios de bienes o servicios por sumas de dinero sin que sea una transacción mercantil (F. Weber, 2008).

Considerando dos formas opuestas de intercambio $\underline{8}$, por un lado, conceptualizamos la transacción mercantil que corresponde a la situación de distancia nula entre dos interacciones. Este tipo de transacción consiste en intercambiar un objeto contra su equivalente (monetario, o bien material, como en el caso del trueque o del pago en especies). Como los participantes aceptan la estricta equivalencia entre los dos objetos intercambiados, y como las dos interacciones (entrega de objeto y contra-transferencia de su pago) son en realidad una sola,

la transacción mercantil concierne a objetos en principio intercambiables (porque se reconoce su equivalencia) y ocurre entre individuos en principio intercambiables (el mismo precio rige sin importar quién sea el cliente). Deja de lado entonces cualquier elemento personal, al que neutraliza, invalida o pone entre paréntesis (Duty y Weber, 2009, p. 71) 
Si la existencia de dinero no es lo que diferencia los tipos de transacciones, lo que caracteriza a aquellas mercantiles es el cálculo, la distancia nula entre las interacciones y que es neutra afectivamente hablando. El bien intercambiado es evaluado independientemente de la relación entre las personas que participan del intercambio, la transferencia es cerrada y afectivamente neutra donde la transferencia y la contra-transferencia se superponen agotando el sentido de la interacción: "La interacción es puntual, sin duración, sin pasado ni futuro; los participantes y los objetos del intercambio son a menudo intercambiables” (F. Weber, 2008, p. 73).

El hecho de que se trate de una interacción que se agota en el acto, sin poner en juego aspectos afectivos, implica un trabajo de "puesta entre paréntesis" de las características personales de los participantes del intercambio (Duty y F. Weber, 2009). Se trata de una abstracción, efectuada, en general, por intermediarios especializados que efectúan la medición física del bien que circula en el mercado o que evalúan su precio.

En el otro extremo de la relación de intercambio, ubica una forma de transacción de tipo recíproca, en la cual existe una distancia entre la transferencia y la contra-transferencia: no se trata de una interacción puntual, sino de una serie de interacciones entre personas que están vinculadas entre sí por una historia de interacciones pasadas que entran en juego en las cosas transferidas (F. Weber, 2008). Un ejemplo típico de estas formas son las transacciones de bienes o servicios puntuales entre familiares, en las cuales el intercambio preciso no es más que un eslabón en una cadena incesante de bienes ofrecidos y servicios prestados, cadena que teje una relación personal con una fuerte carga afectiva, construida sobre la base de afectos, rencores, preocupaciones, recriminaciones y gratitud (F. Weber, 2008).

Estas formas de reciprocidad $y$ valoraciones individuales $\mathrm{o}$ familiares no se sustentan exclusivamente en cuestiones de solidaridad, sino que incluyen negociaciones en torno a diversas valoraciones $y$, en general, en las estimaciones de confiabilidad entran en juego relaciones de poder, diferenciaciones de clase y género.

Cabe señalar que también en las transacciones mercantiles basadas en el cálculo, donde supuestamente se prescindiría de los “ruidos” característicos de relaciones sociales (teñidas de emociones y viciadas por las particulares combinaciones de historias compartidas y de desencuentros, producto de la interacción cotidiana), en los hechos conllevan formas de predicción y valoración imbricadas en circuitos de significado, en los que no pueden quedar fuera dimensiones sociales, culturales e incluso éticas (Villareal, 2008).

Considerar separadamente la cuestión del medio de pago respecto de la transacción en sí, nos permite reflexionar independientemente sobre el tipo de interacción (mercantil o reciproca) y la presencia de transferencias monetarias en la transacción. Como señalamos anteriormente, lejos de determinar la naturaleza de las interacciones, los medios de intercambio incorporados (incluida la moneda legal), asumen connotaciones particulares. Los participantes de las interacciones ajustan de forma particular los medios de intercambio para establecer distinciones entre los diferentes tipos de relaciones sociales (Wilkis, 2013; Zelizer, 2011).

En relación a las formas en que se dan estos pagos, Zelizer (2009) plantea que no debemos atender (a la hora de analizar las relaciones sociales en la esfera económica) solo a las explicaciones 
puramente "eficientistas" de los sistemas de pago. Las medidas efectivas de desempeño o las estructuras de recompensas no logran dar cuenta de la creación, aceptación cambiante y diversidad de arreglos que implican. En el mundo económico (sobre todo laboral) existen compensaciones que generan distinciones y diferenciaciones que sirven para crear y mantener diferentes grupos de relaciones sociales, y que se corresponden con significados distintos. Las formas de compensación pueden servir para supervisar y regular la productividad de los trabajadores, asegurar la lealtad, establecer diferencias entre los trabajadores, reconocer saberes, generar jerarquías, entre otros:

En discusión estaban no sólo el monto y el carácter del pago, sino también las relaciones sociales apropiadas entre empleador y empleado. No sólo los bonos, sino también las comisiones, los premios, las cuentas de gastos, los autos de la compañía, las millas de viajero frecuente, los beneficios en salud y aún la llave para acceder a los baños de los ejecutivos se transforman en pagos resistidos pero cruciales, contingentes, discrecionales que definen relaciones entre las personas en el interior de las firmas contemporáneas. Ellos anuncian, y hasta cierto punto determinan, qué pares de trabajadores son iguales o desiguales, cercanos o distantes, solidarios 0 competitivos (Zelizer, 2009, p. 50).

En un sentido similar, Quaranta (2007) señala que los sistemas de remuneración pueden funcionar como un dispositivo de incentivo o de control: la función del pago a destajo puede ser una forma de incrementar la productividad física del trabajo; la inclusión de formas de pago según resultados constituye una modalidad de involucrar a los trabajadores; la combinación del salario con compensaciones monetarias y no monetarias, en el marco de las relaciones personales que establecen patrones y trabajadores, contribuyen a la conformación de trabajadores comprometidos con el proceso productivo y sus resultados, e implican una forma de identificación basada en lazos personales que favorecen la desvinculación de los comportamientos de los actores sociales de su posición de clase (Quaranta, 2007).

Entonces, a la cuestión de los intercambios que fluyen entre estas relaciones sociales proponemos conceptualizarla a partir de la caracterización de la forma que asumen (mercantiles o recíprocas) y de la definición de Florence Weber, destacando que las relaciones no son simétricas y armónicas sino que están atravesadas por cuestiones de clase, género, relaciones de poder y conflictos. En cada una de las relaciones sociales que establecen los empresarios con los otros actores se puede indagar, además, qué formas adquieren las remuneraciones, si estas establecen distinciones dentro del conjunto de las relaciones, y si se han transformado recientemente, por qué y en qué sentido; cómo se calcula, qué se toma en cuenta para medir el pago; qué medios de pago se usan y qué significan; como así también qué distinciones introducen en las relaciones estos medios de pago.

\subsection{Tensión entre lógicas opuestas de estructurar las relaciones sociales}

Luego de haber revisado las dimensiones con las que proponemos describir las relaciones sociales en el trabajo agropecuario, consideramos finalmente la tensión entre lógicas opuestas de estructurarlas. Para este objetivo tomaremos el concepto de racionalidad de Max Weber, entendido como lógica que ordena la conducta bajo modelos generales de acción, el principio orientador de la acción individual y social. Si bien el análisis que realizaremos no logrará abarcar la complejidad del estudio de la racionalidad, nos interesa recuperar la tensión entre lógicas diferentes de desarrollar 
las relaciones sociales.

En la obra de Weber se encuentran distintas acepciones del concepto de racionalidad, y aplicadas a distintas áreas como dominación, ley, economía, religión, música y ciencia (Eisen, 1978). Sin embargo, en el análisis sociológico de la vida económica en Economía y Sociedad (Weber, 2012[1922]), luego de formular lo que considera una “acción económica racional”, realiza una distinción central para el análisis posterior del capitalismo (y sus características intrínsecas): la diferenciación entre racionalidad material y racionalidad formal, que en conexión explican el funcionamiento del sistema económico.

La racionalidad formal a la que refiere se trata de un concepto que expresa el "grado de cálculo que le es técnicamente posible y que aplica realmente en una gestión económica”, que coincide con el mayor grado de calculabilidad (cuyo mayor desarrollo es alcanzado con el cálculo en dinero y del capital, forma más perfeccionada del mismo) (Weber, 2012[1922]). En cambio, la racionalidad material expresa el grado en que el abastecimiento de bienes dentro de un grupo humano tiene lugar por medio de una acción social económicamente orientada según ciertos postulados de valor (cualquiera sea su clase), de manera que esa acción pueda ser considerada desde la perspectiva de tales postulados valorativos. Es decir, la racionalidad material implica el planteamiento de exigencias extraeconómicas, en función de las cuales la acción económica resulta orientada con arreglo a valores o fines materiales. La amistad, por ejemplo, siempre y cuando se adhiera a ciertos valores como lealtad, compasión y asistencia mutua, constituye una racionalidad sustancial (Kalberg, 1980). En este marco, el actor actúa según “mandatos” o de acuerdo con "exigencias” que cree dirigidos a él (y frente a los cuales se siente obligado) (Weber, 2012[1922]). Como señala Brubarker (citado en Kalberg, 1980), la diferenciación entre racionalidad formal y sustancial puede ser interpretada como una tensión entre valores conflictivos: entre el cálculo, la eficiencia y la impersonalidad por un lado, y la fraternidad, la igualdad y la caridad por el otro.

En el planteo de Weber, cuanto más avanza el mundo de la economía capitalista en sus propias leyes internas, tanto más se desestructuran otras lógicas de relaciones. El avance del capitalismo implica la plena calculabilidad y previsibilidad del mundo, de dominación de todos los fenómenos mediante el cálculo. Esta se halla en la base de la economía puramente monetaria que da por resultado "de una parte, la calculabilidad objetiva del rendimiento lucrativo del individuo y su gasto, y le abre, por otra parte -en virtud del desarrollo del 'cambio indirecto' que permite el dinero-, la posibilidad de satisfacer libremente necesidades individuales” (Weber, 2012[1922], p. 308).

La introducción del cálculo racional en las relaciones sociales conlleva a la distinción entre una esfera doméstica (ámbito de la familiaridad) y una esfera propiamente económica (ámbito del trabajo), y resulta condición de posibilidad para la producción capitalista (Weber, 2012[1922]). La transformación de las relaciones sociales en el marco del avance del capitalismo se traduce en una modalidad más impersonal y "formal”. Las relaciones formales implican que la conducta reciproca se determina y establece mediante reglas fijas. Las relaciones surgen de forma planificada de una acción u organización orientada a fines. Se relaciona con la burocratización en tanto aumenta el grado de formalización de las relaciones sociales. Las relaciones formales están restrictivamente reguladas de forma directa por leyes, contratos, preceptos, permisos, obligaciones, normas de conducta ceremoniosa (reglas de etiqueta, modales), así como por sanciones. Desde el punto de 
vista del cumplimiento de las reglas, están sujetas a una supervisión y a un control extremo. Gracias al ordenamiento formal de las relaciones, aumenta la seguridad en las expectativas mutuas y la previsibilidad de las acciones de quienes ocupan o desempeñan roles recíprocamente relacionados. Estas relaciones carecen de aspectos emocionales y subjetivos, y facilitan a los interesados distancia interna y, al mismo tiempo, adaptación externa (Heinz Hillmann, 1994, p. 771).

En el plano de las relaciones en el mundo del trabajo, el avance del capitalismo implica, en la visión de Weber, racionalización total de la organización del trabajo, la deshumanización, la calculabilidad y la mecanización metódica. Las relaciones laborales adoptan un carácter puramente técnico, sometidas a las exigencias del cálculo de rentabilidad del capital (Kohler y Martín, 2005). Las relaciones laborales burocráticas son relaciones dominadas por la objetividad de la organizacional racional, representada no por la persona de un jefe, sino por un especialista profesional que supervisa el orden formal y las reglas generales sin ningún tipo de emoción y sensibilidad personal. Se trata de la subordinación del ejercicio del mando a normas abstractas (Kohler y Martín, 2005).

Respecto a formas opuestas de racionalidad, en el estudio sobre la transformación del modo de vida chacarero, Balsa (2006) destaca la complejidad del análisis de la misma en los productores pampeanos, siendo que no puede ser caracterizada como estrictamente formal: si bien atiende al cálculo, también otros postulados (asociados a la unidad domestica) le imponen limites y objetivos.

Mas pareciera prevalecer una situación intermedia, que combina cierta racionalidad formal orientada hacia la maximización de los beneficios, pero limitada por algunos objetivos familiares, como el mantener un nivel de vida de la clase media-alta urbana y el de enviar los hijos a la universidad, que pueden conspirar contra la mejor reinversión de los beneficios, según un cálculo meramente formal (Balsa, 2006, p. 258).

El cambio en los modos de vida, asociada a la residencia urbana, pareciera implicar tipos de racionalidades más de tipo formal, aunque, como señala el autor, en muchos casos siguió habiendo objetivos materiales por encima de los beneficios (Balsa, 2006).

En nuestro trabajo consideramos, a partir de las categorías weberianas, formas diferentes de estructurar las relaciones sociales a partir de lógicas opuestas, y situaciones intermedias que se acercan en algunos aspectos a unas u otras. También recuperamos la tensión planteada en Weber respecto a si existe una forma de estructurar las relaciones que cobra más relevancia por sobre otras, y que implica una separación de las esferas familiares y personales de las laborales. Estas categorías nos permiten analizar si las relaciones se estructuran cada vez más sobre la base de la calculabilidad; es decir, si las transformaciones en la producción, en un sentido más capitalista, implican que las relaciones sociales en el trabajo agropecuario se vuelvan más impersonales, asentadas en lo técnico y tecnológico, sometidas a las exigencias del cálculo de rentabilidad del capital. Además, indagar si existe una tendencia hacia la formalización de las relaciones sociales y, en detalle, qué aspectos se formalizan, si implican una mayor distancia entre los actores; o si existe una persistencia de relaciones basadas en postulados de valor, como la amistad, lo familiar, las formas heredadas, por ejemplo; o se presentan relaciones sociales que combinan aspectos de ambos 
tipos de lógicas.

\section{REFLEXIONES FINALES}

\section{Nuestra propuesta conceptual}

Considerando en conjunto las diferentes perspectivas y categorías junto a lo relevado en el trabajo de campo, identificamos unidades productivas en las que en las relaciones se presentan extremos de cada una de las dimensiones. Teniendo en cuentas los ejes articuladores de cada una de estas formas, a modo de síntesis, las reconstruiremos bajo una denominación.

Por un lado, conceptualizamos la existencia de relaciones personales. En las mismas se dan explotaciones en las que las relaciones sociales se anclan sobre las personas concretas, se construyen en base al tiempo compartido en los espacios locales (previo o en el sitio de trabajo), y que mantienen una tradición (un modo de hacer las cosas “como siempre se han hecho”). La confianza se basa en la persona concreta, en los "compromisos personales", y esto implica que no se recurra a mecanismos de supervisión del trabajo y puedan delegar el "hacer” cotidiano. Los intercambios están regidos, además de cierta referencia con respecto al mercado, fundamentalmente en la consideración de la persona concreta del trabajador, en la confianza que le tiene el empleador y en lo que representa para la empresa (gravitando en las formas de remuneración la historia compartida). Ponerle una denominación a este tipo de relación no resulta tarea sencilla, pero hemos optado por llamarlas relaciones personales porque consideramos que lo personal es una etiqueta que identifica (aunque no de modo exhaustivo) un componente predominante de las relaciones. Cabe aclarar que el hecho de que las consideremos personales no significa que el cálculo, el interés y el lucro no estén presentes.

Por su parte, conceptualizamos la existencia de relaciones mercantiles en explotaciones en las que las relaciones sociales se anclan en otros soportes diferentes a los tradicionales, fundamentalmente en los sistemas expertos y en la organización racional del trabajo. En estas relaciones las personas concretas tienen relevancia, pero la estructura se basa en los roles estipulados por la organización del trabajo, las capacidades y saberes necesarios para el desarrollo de un puesto laboral. Las mismas se desarrollan mediadas por una estructura de supervisión de las tareas cotidianas. Los intercambios se estructuran a partir de los parámetros de mercado y del cálculo en base al interés económico. Las formas de remuneración buscan maximizar el rendimiento productivo. A estas relaciones las denominaremos mercantiles, porque aunque las personas concretas se conocen, pueden tener una trayectoria compartida, etc., este conocimiento no resulta predominante para estructurar la relación, y las personas pueden ser reemplazadas por otras si no resultan convenientes para el desarrollo de la producción.

Los distintos tipos de relaciones sociales implican una forma de racionalidad que incluye otras dimensiones que no consideramos, a la par que involucra formas opuestas "puras" que no describen completamente a nuestros casos, pero que resultan útiles para comenzar el análisis. En cada uno de los grupos de productores que entrevistamos la cuestión del cálculo está presente como aspecto relevante para estructurar las relaciones, a pesar de que el afecto y la confianza resulten estructuradores de las relaciones. En otro grupo de empresarios relevados, el interés y el cálculo son los principios orientadores de la relación con otros actores, y sin embargo también se entrecruza con 
cuestiones personales, con prácticas no estrictamente mercantiles, etc. Entre los extremos de relaciones sociales, encontramos a la mayoría de los casos que en cierta medida comparten características con uno u otro polo, conformando un “continuo” en el que lo personal y lo mercantil se entrecruzan configurando "nuevos tipos” de articulaciones sociales con una lógica propia.

\section{Notas}

1 "Cada vez que aparecemos en los medios es porque algo hizo ruido. Tenemos que tratar de que se hable favorablemente y que se quiera al campo". Trasmonte, Daniel (2017). Entrevista, Revista Crea, 435. Buenos Aires: AACREA.

$\underline{2}$ El trabajo al que hacemos referencia es en el marco de la Beca Posgrado CONICET, ha sido presentado en forma de tesis en la Maestría en Estudios Sociales Agrarios de FLACSO, y continua profundizándose en el Doctorado en Ciencias Sociales de la UNQ. El trabajo empírico se realizó en tres partidos de la provincia de Buenos Aires (Junín, Mar Chiquita y Pehuajó) en los cuales, mediante entrevistas a productores agropecuarios de diverso perfil, se abordaron las características de las relaciones sociales bajo las cuales desarrollan la producción.

33Según Saltalamacchia, las teorías tienen dos papeles diferentes en un proceso de investigación: una función explicativa, que presenta una imagen acabada del hecho, o una función heurística que permite abrir la percepción hacia ciertos campos de investigación (Saltalamacchia, 1997).

4 El estudio, que analiza sobre todo los procesos de regulación social del trabajo en la horticultura, establece como dimensiones de análisis la cuestión socio institucional, los trabajadores y sus trayectorias, la organización y contratación del trabajo (producciones, división del trabajo, supervisión, contratación, intermediación, redes sociales, etc.), relaciones de trabajo en el sitio de producción (construcción de consensos, involucramiento de los trabajadores, emergencia de conflictos, relaciones de reciprocidad, etc.), sistemas y niveles de remuneración (formas y tipos). (Quaranta, 2007).

$\underline{5}$ Estudios sobre el agro argentino que utilizan el concepto de desanclaje de las relaciones sociales para caracterizar los tipos de actores del sector y sus transformaciones pueden verse en Gras y Hernández (2009, 2013).

$\underline{6}$ Giddens le asigna un lugar destacado a las relaciones de amistad en el mundo premoderno, en el cual las amistades siempre estaban sujetas a ser puestas al servicio en diversas situaciones en los que los lazos comunitarios o de parentesco resultaban insuficientes, como por ejemplo alianzas económicas, ayuda en determinadas actividades, etc.

$\underline{7}$ Los dos complejos institucionales significativos en el desarrollo de la modernidad son, según Giddens, el Estado Nación y la producción capitalista sistemática, con la completa mercantilización de los productos y del trabajo asalariado; no se dan en anteriores periodos históricos, y ambos han mantenido una autonomía al margen de las tendencias del desarrollo global.

$\underline{8}$ Esta diferenciación se encuadra en la oposición, desde la antropología económica, de dones y mercado. Si bien se ha abordado como dos formas antagónicas, existen otras perspectivas que, 
combinando diferentes lecturas de Marcel Mauss, plantean una lectura del don no como antimercado (F. Weber, 2008). Florence Weber combina lecturas francesas del trabajo de Mauss que ponen el acento en diferentes dimensiones para dar cuenta de tres articulaciones de las formas en que se dan los lazos y los intercambios de cosas que entre ellos fluyen.

\section{BIBLIOGRAFIA}

Albadalejo, C. (2013). Dinámica de la inserción territorial de la agricultura pampeana y la emergencia del agrobussiness. En Gras C. y Hernández (comp.), El agro como negocio. Buenos Aires: Biblos.

Anlló, G., Bisang, R. y M. Campi (2013). El modelo de organización de la producción agrícola: de la integración vertical a la agricultura en red. En G. Anlló, R. Bisang y M. Campi (coord.), Claves para repensar el agro argentino. Buenos Aires: Eudeba.

Balsa, J. (2006). El desvanecimiento del mundo chacarero. Bernal: UNQ Ediciones.

Barsky, O. y Gelman, J. (2009). Historia del agro, Argentino. Buenos Aires: Sudamérica.

Bertaux, D. (1988). El enfoque biográfico: su validez metodológica, sus potencialidades. En Historia oral e historias de vida, Cuadernos de Ciencias Sociales, $\mathrm{n}^{\circ}$ 18. Costa Rica: FLACSO.

Bertaux, D. (1993). De la perspectiva de la historia de vida a la transformación de la práctica sociológica. En Marina, C. (comp.), La historia de vida: método y experiencia. Madrid: Ed. Debate.

Bisang, R.y Kosacoff, B. (2006). Redes de producción en el agro pampeano. Presentado en XIV Congreso Anual AAPRESID, Rosario, Argentina.

Bisang, R., Anlló, G. y Camp, M. (2008). Una revolución (no tan) silenciosa. Claves para pensar el agro en la Argentina. En Desarrollo Económico No 190-191. Buenos Aires: IDES.

Bourdieu, P. (2008) [1973]. El Oficio del Sociólogo. Argentina: Siglo XXI Editores.

De Grande, P. (2007). Lazos de familia en el análisis de las redes personales en Argentina. IX Jornadas Argentinas de Estudios de Población, Asociación de Estudios de Población de la Argentina (AEPA), Huerta Grande. Recuperado de https://www.aacademica.org/pablo.de.grande/4

Dufy, C. y Weber F. (2009). Más allá de la gran división. Sociología, economía y etnografía. Buenos Aires: Antropofagia.

Eisen, A. (1978). The Meanings and Confusions of Weberian'Rationality'. The British Journal of Sociology, 29(1), 57-70.

Eisenhardt, K. (1989). Building theory from Case Study Research. The Academy of Managment Review, 14(4). 
Giarracca, N. y Teubal, M. (2008). Del desarrollo agroindustrial a la expansión del 'agronegocio’: el caso argentino. En Bernardo Mançano Fernandes (org.), Campesinato e agronegócio na América Latina: a questao agrária actual. Sao Paulo: CLACSO.

Giarracca, N. y Teubal, M. (2010). Del paro agrario a las elecciones de 2009. Tramas, reflexiones y debates. Buenos Aires: Antropofagia.

Giberti, H. (2008). La cuestión agraria en Argentina. Revista Mundo Agrario, 8(16).

Giddens, A. (1993). Consecuencias de la modernidad. Madrid: Alianza.

Giddens, A. (1995). Modernidad e identidad del yo. El yo y la sociedad en la época contemporánea. Barcelona: Ed. Península.

Giddens, A. (1997). Vivir en una sociedad postradicional. En Beck, U., Giddens, A. y Lash, S., Modernización reflexiva. Política, tradición y estética en el orden social moderno. Madrid: Alianza.

Granovetter, M. (1973). The strength of weak ties. American Journal of Sociology, 78(6), 1360 1380 [Edición en Castellano: (2000). La Fuerza de los vínculos débiles. Política y sociedad, 33. Universidad Complutense de Madrid, Traducción de María Ángeles García Verdasco.]

Gras, C. y Hernández, V. (2009). El fenómeno sojero en perspectiva: dimensiones productivas, sociales y simbólicas de la globalización agrorural en la Argentina. En Gras, C. y Hernandez, V. (coords), La Argentina rural. De la Agricultura familiar a los agronegocios. Buenos Aires: Editorial Biblos. Gras, C. y Hernández, V. 2013). El agro como negocio. Buenos Aires: Editorial Biblos.

Gutierréz, A. (2007). Redes e intercambio de capitales en condiciones de pobreza: dimensión relacional y dimensión vincular. Presentado en Ier. Reunión Latinoamericana de Análisis de Redes Sociales, UNLP, La Plata, Argentina.

Hillmann, H. (1994). Diccionario enciclopédico de sociología. Barcelona: Herder editorial.

Kalberg, S. (1980). Max Weber's Types of Rationality: Cornerstones for the Analysis of Rationalization Processes. The American Journal of Sociology, 85(5), 1145-1179. Recuperado de: http://www.jstor.org/stable/2778894

Kohler, H-D. y Martín, A. (2005). Manual de la Sociología del Trabajo y de las Relaciones Laborales. Madrid: Delta Publicaciones.

Luhmann, N. (1996). Confianza. España: Ed. Anthropos.

Newby, H. (1983). Capítulo 4. En Newby, H. y Sevilla Guzmán, E., Introducción a la sociología rural. Madrid: Alianza Universidad.

Pucci, F. et al. (2006). Confianza, instituciones y organización del trabajo. Ponencia presentada al IV Seminario Anual de Investigadores del Departamento de Sociología de la Facultad de Ciencias Sociales, UDELAR.

Quaranta, G. (2007). Reestructuración y organización social del trabajo en producciones agrarias de la región pampeana argentina (Tesis para optar por el grado de Doctor). Instituto de Sociología y 
Estudios Campesinos, Universidad de Córdoba, Córdoba.

Saltalamacchia, H. (1994). Historia de vida y reconstrucción articulada: reflexiones teóricometodológicas a partir de una experiencia de investigación. Anthropos, Suplemento 45 (Círculos de reflexión latinoamericana en Ciencias Sociales)..

Saltalamacchia, H. (1997). Los datos y su creación. Kryteria, Puerto Rico: Caguas.

Sili, M. (2005). La Argentina rural. De la crisis de la modernización agraria a la construcción de un nuevo paradigma de desarrollo de los territorios rurales. Buenos Aires: INTA.

Sili, M. y Bustos Cara, R. (1996). Modernización rural y diferenciación social en la Pampa Argentina. Publicado en Actas Primer Congreso de Latinoamericanistas, Salamanca, España.

Villareal, M. (2008). Sacando cuentas: Prácticas financieras y marcos de calculabilidad en el México rural. Revista Crítica en Desarrollo, 2, 131-149.

Weber, F. (2008). Transacciones económicas y relaciones personales. Una etnografía económica después de la gran división. Revista Crítica en Desarrollo, 2.

Weber, M. (2012[1922]). Economía y sociedad, esbozo de sociología comprensiva. México: FCE.

Wilkis, A. (2013). Las sospechas del dinero. Moral y economía en la vida popular. Buenos Aires: Paidos.

Zelizer, V. (2009). La negociación de la intimidad. Buenos Aires: Fondo de Cultura.

Zelizer, V. (2011). El significado social del dinero. Buenos Aires: Fondo de Cultura. 